\section{The OPTIMISE data project: toward improving multiple sclerosis treatment}

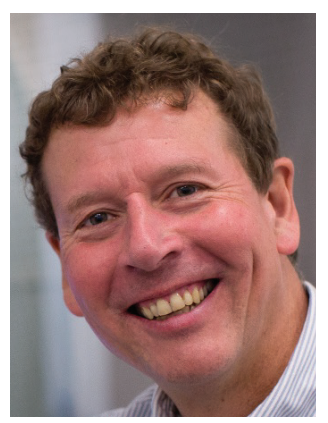

Paul M Matthews* speaks to Ellen Clarke, Commissioning Editor: Paul Matthews, OBE, MD, DPhil, FRCP, FMedSci is Head of the new Division of Brain Sciences at Imperial College, London. He was the founding Director of two leading research imaging centers, the University of Oxford Centre for Functional Magnetic Resonance Imaging of the Brain (FMRIB) and of GlaxoSmithKline's Clinical Imaging Centre. From 2005 to 2014 he was Vice President of GlaxoSmithKline Medicines Discovery and Development. Professor Matthews' research has extended applications of advanced imaging methods to answer a new spectrum of clinical research questions. He worked with Oxford colleagues in applications developments for advanced structural and functional brain imaging incorporated into the open access FSL software distributed by the FMRIB Centre, now one of the two most widely available image analysis software 'toolboxes' worldwide. While at Imperial and GSK, his group piloted approaches extending these methods for the first controlled, prospectively designed imaging genetics studies. He has been Chair of the Imaging Working Group for UK Biobank over the last 5 years, which is pioneering an ambitious program for very large population imaging as part of the UK Biobank. MRI scanning of the brain, heart and body, along with DEXA and 3D carotid ultrasound, was initiated in an imaging center at UK Biobank's Cheadle site in May 2014. A focus has been to address the challenge of neurodegeneration in multiple sclerosis and enhancing intrinsic brain repair and plasticity for functional recovery. More recent collaborative work with Imanova Ltd builds on studies of the genetics and pharmacology of the latest generation of PET microglial imaging agents to relate microglial activation and neurodegeneration in vivo. Professor Matthews received an OBE in 2008 for services to neuroscience. He was elected to the Academy of Medical Sciences in 2014.

\section{Q How did you first become involved in the field of clinical brain imaging?}

I started work in the related field of magnetic resonance spectroscopy while a graduate student at Oxford, when clinical MRI was just developing as a field. My first use of MRI was during my early medical training, but I rapidly moved toward research applications during my time as a neurology registrar, when I started using brain MRI to provide a more specific phenotype for patients with unusual neurological syndromes. With a move to Oxford to establish the Centre for Functional Magnetic Resonance Imaging in 1995, a singular effort to exploit brain MRI began. Initially, we focused strictly on functional studies using BOLD imaging techniques. However, by the beginning of this last decade, the group began to address more precise ways of measuring brain size and shape and its connectivity. In 2005, I moved to GlaxoSmithKline to lead development of their Clinical Imaging Centre. While the bulk of the activity concerned brain imaging, I found myself working on a much broader range of imaging projects as appreciation of

*Division of Brain Sciences, Department of Medicine, Imperial College, London E515, Burlington Danes, Hammersmith Hospital, DuCane Road, London, W12 0NN, UK; p.matthews@imperial.ac.uk

\section{Future}

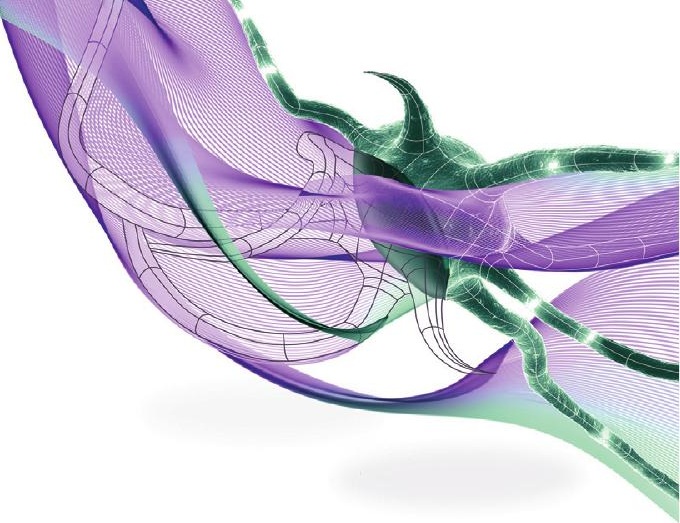


the potential of MRI grew across other therapeutic areas. Coming back to academia and Imperial, I am again addressing only problems of the brain and have a special interest in using imaging to discover the role that inflammation plays in neurodegeneration at different times in the course of multiple sclerosis and dementias.

\section{Q What would you say have been your most significant contributions to neuroscience to date?}

'Significant' may be too challenging a word! As a scientist, I am always trying to think beyond where I am and never satisfied that I have reached deeply enough into nature to have 'solved' a problem in a significant way. Let me restate the question as, 'What are the most satisfying contributions that you have made?' As a graduate student, I worked with Keith Thulborn and John Waterton, both excellent scientists and teachers, in characterizing the basic phenomenon underlying what came to be known as BOLD contrast after Ogawa's much later, brilliant studies. While in Montreal, Doug Arnold and I recognized that axonal loss was a major aspect of the pathology of MS, work that I validated with Margaret Esiri and colleagues after my move to Oxford. It was in Oxford that I also provided evidence for the generalizability of concepts of functional adaptation of the brain as a mechanism for brain plasticity and where, building on the hugely insightful theoretical work of Tim Behrens, my younger colleagues and I developed a robust approach to brain connectivity based parcellation of grey matter. GSK led me to develop an interest in PET. With my close colleagues, Ilan Rabiner and Roger Gunn, and Ed Bullmore from Cambridge, we developed robust demonstrations of the specific relationships between modulatory receptor activity and changes in specific systems level function. Linking molecular changes to brain systems level function has been where I was trying to go through my career!

Q What are the main aims of the OPTIMISE project \& how do you hope to achieve these? Although many individually held clinical research databases have been developed over the last few decades, access to them is limited, data currently are acquired in different ways and differences in definitions and indexing and software platforms preclude direct integration. MS experts, charity leads, people with MS and industry representatives have recognized that a key enabler for improved research and care would be a flexibly configured, secure IT framework and associated IT tools for capture of clinical, patient-centered, genomic, biomarker and imaging data that would build on an agreed ontology for multiple sclerosis.

We are developing the OPTIMISE Portal, as a first open-source, secure, high capacity clinical, patient-centered, laboratory and imaging data acquisition, storage and analysis system with sufficient robustness for widespread adoption. The software infrastructure for the OPTIMISE is to be based on the eTRIKS platform. The eTRIKS infrastructure is intended to give the OPTIMISE Portal the robustness that is needed to become an international standard in open-source software for management of MS data (and to facilitate extension to other applications, e.g., dementia). Of particular salience for the design needs of the OPTIMISE Portal is that eTRIKS is an open system designed to enable ready extensibility by interfacing with other components to form a rich informatics infrastructure provided by researchers using eTRIKS across the UK and Europe. An exciting extension of the OPTIMISE Portal will involve extension of applications of smartphone apps to capture GPS data and capturing of movement, heart rate and brain function data with small remote sensors.

Finally, OPTIMISE is designed with a broad range of users in mind: doctors, researchers and patients. The platform is intended to enhance control of data by those who submit it. This will enhance trust and the range of applications possible. We don't see it just as facilitating advanced research. It also should be able to help people with MS understand their own condition better.

\section{Q What are your roles \& responsibilities in the project?}

I act as the Principal Investigator, which means Chief Executive and Chief Financial Officer. My first role is to keep the science firmly in mind as I ensure that this complex project moves toward completion. A second task that will grow in importance as the project matures is to ensure that a broad range of stakeholders is involved to help the project deliver real value to the community - which includes people with MS, clinicians and researchers.

\section{Q Will there be new technologies implemented in the project?}

OPTIMISE involves a close collaboration between the Imperial College London Faculty 
of Medicine and the College Data Science Institute, directed by Professor Yike Guo. The project involves the integration of recently developed or evolving platforms for data management in a configuration that is tailored specifically for applications in multiple sclerosis. It will involve development of new tools for capturing data and incentivising data contributions from people with MS by ensuring that they benefit from the exercise directly. New technologies are being implemented for 'decoding' the signals from movement sensors to provide more quantitative measures of walking. We also are working on novel technologies to enable simple, home EEG monitoring over extended times to allow us to better investigate pathologies of sleep in MS, which may be contributing to a number of symptoms.

\section{Q What different types of data will be collected from patients in order to meet the aims of the project?}

The clinician interface will collect standard clinical history and patient performance data concerned with patients' thinking, vision, walking and motor control, balance, sensation and bowel and bladder functions. Information on medicines used and any adverse events associated with them, as well as doctor or hospital visits, will be recorded. An important feature is that doctors also will be able to store imaging and laboratory test data, including genetics and more advanced 'omics. The interface for people with MS will allow them to record information about how they feel and are functioning in their daily life. We also hope to be able to administer simple tests to better assess thinking and memory. A sensor portal will be able to capture data from gait sensors and, we hope, additional EEG or GPS data.

\section{Q How important is the open access nature of the project?}

Open access to the platform is very important. Our objective is to create a tool for the community that will lower the barriers to the organized collection of data on people with MS, how they change over time and how they respond to medicines. Wider use of a common platform that uses widely accepted data management standards will make the sharing of data for the benefit of people with MS (e.g., for understanding what kinds of people benefit most from which medicines) easier. However, although the platform will be open access, a key feature is that each data donor
- whether a person with MS or a doctor - will be able to decide who they want to share data with and when.

\section{Q What is the scale of the project? Are there future plans for expansion?}

Currently, this project is planned for a 3-year period. We intend to build the platform, pilot and then refine it and, finally, establish a way of sharing it with the broader community. This could involve, for example, working closely with important data initiatives in the field such as the UK MS Registry. With initial success of the platform, developments for the future likely would include increasing the range of tools available in OPTIMISE for data analysis and adding novel kinds of information from new forms of remote sensors.

\section{Q What major challenges are there currently in the field of multiple sclerosis research?}

The field is still lacking a well-accepted, unitary model for the genesis and evolution of the disease through its long course. What causes the disease to start? What controls disease activity? What are the mechanisms underlying disease progression? These are not challenges that can be addressed with either small datasets local to a single institution or data collected for short periods of time. Comprehensive data on large numbers of people with MS, collected over decades and able to be aggregated meaningfully is needed. From this, we need to move from clinical expression to understanding systems involvement and specific molecular drivers that could become drug targets.

\section{Q Looking forward, what advances do you think are likely to occur in the field during the next five years? How might the project contribute to these advances?}

The 'low hanging fruit' will be patient stratification - defining sets of patient characteristics that predict prognosis and, possibly, response to medicines. There also is a big potential for defining ways of better monitoring medicine responsiveness early, so doctors can get the right medicine to the right patient quickly! In the longer term, there is an opportunity for improving our efforts in pharmacovigilance (identifying adverse events associated with medicines). The 'holy grail' will be improving understanding of the relationship between molecular factors and 
INTERVIEW Matthews

clinical course in ways that allow us to define mechanisms of disease that can lead to new insights for management.

\section{Disclaimer}

The opinions expressed in this interview are those of the interviewee and do not necessarily reflect the views of Future Medicine Ltd.

Financial \& competing interests disclosure

PM Matthews gratefully acknowledges personal support from the Edmind J Safra Foundation and Lilly Safra and from the Imperial College NIHR Biomedical Research
Center. He has received honoraria or research support from GSK, Biogen IDEC, Novartis, IXICO, the EC, the Progressive MS Alliance, the MS Society of Great Britain and Northern Ireland and the MRC. He has received attendance fees from CIHR, Wellcome Trust, Sir Jules Thorne Trust, Finnish Academy of Science, WWTF and HEFCE. The author has no other relevant affliations or financial involvement with any organization or entity with a financial interest in or financial conflict with the subject matter or materials discussed in the manuscript apart from those disclosed.

No writing assistance was utilized in the production of this manuscript. 